ARTICLE

\title{
Perception of a divergent family of phytocytokines by the Arabidopsis receptor kinase MIK2
}

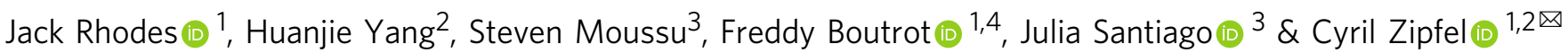

Plant genomes encode hundreds of receptor kinases and peptides, but the number of known plant receptor-ligand pairs is limited. We report that the Arabidopsis leucine-rich repeat receptor kinase LRR-RK MALE DISCOVERER 1-INTERACTING RECEPTOR LIKE KINASE 2 (MIK2) is the receptor for the SERINE RICH ENDOGENOUS PEPTIDE (SCOOP) phytocytokines. MIK2 is necessary and sufficient for immune responses triggered by multiple SCOOP peptides, suggesting that MIK2 is the receptor for this divergent family of peptides. Accordingly, the SCOOP12 peptide directly binds MIK2 and triggers complex formation between MIK2 and the BRASSINOSTEROID INSENSITIVE 1-ASSOCIATED KINASE 1 (BAK1) co-receptor. MIK2 is required for resistance to the important root pathogen Fusarium oxysporum. Notably, we reveal that Fusarium proteomes encode SCOOP-like sequences, and corresponding synthetic peptides induce MIK2-dependent immune responses. These results suggest that MIK2 may recognise Fusarium-derived SCOOP-like sequences to induce immunity against Fusarium. The definition of SCOOPs as MIK2 ligands will help to unravel the multiple roles played by MIK2 during plant growth, development and stress responses.

\footnotetext{
${ }^{1}$ The Sainsbury Laboratory, University of East Anglia, Norwich Research Park, Norwich, UK. ${ }^{2}$ Institute of Plant and Microbial Biology, Zurich-Basel Plant Science Center, University of Zurich, Zurich, Switzerland. ${ }^{3}$ The Plant Signaling Mechanisms Laboratory, Department of Plant Molecular Biology, University of Lausanne, Lausanne, Switzerland. ${ }^{4}$ Present address: Anova-Plus, Évry, Évry-Courcouronnes, France. ${ }^{\bowtie}$ email: cyril.zipfel@botinst.uzh.ch
} 
$\mathrm{P}$ lants employ cell-surface localized receptors, such as receptor kinases (RKs), to sense their extracellular environment and coordinate their growth and development in response to endogenous and exogenous cues ${ }^{1}$. Secreted plant peptides-encoded by hundreds of genes in plant genomes-have recently emerged as playing a major role in this autocrine and paracrine communication and are proposed as a new class of plant hormones ${ }^{2}$. The number of known receptors for such peptides however remains scarce.

The leucine-rich repeat receptor kinase (LRR-RK) MALE DISCOVERER 1-INTERACTING RECEPTOR-LIKE KINASE 2 (MIK2) was initially identified for its proposed role in pollen tube guidance mediated by the cysteine-rich AtLURE1 peptides. In this context, it was shown to interact with the LRR-RK MALE DISCOVERER 1 (MDIS1), and was therefore proposed to be part of a receptor complex for AtLURE1 peptides ${ }^{3,4}$. At the same time, another set of LRR-RKs, POLLEN-SPECIFIC RECEPTOR-LIKE KINASE 6 (PRK6) and related proteins, were shown to be involved in AtLURE1 perception ${ }^{5}$. Structural and biochemical work then showed that AtLURE1 peptides bind to PRK6, but not to MIK2 ${ }^{6}$, and the genetic role of PRK6 in AtLURE1 perception was recently corroborated $^{7}$. These findings questioned whether AtLURE1s are ligands for a potential MDIS1-MIK2 complex. Accordingly, it was later revealed that MIK2 (also named LEUCINE-RICH REPEAT KINASE FAMILY PROTEIN INDUCED BY SALT STRESS, or LRR-KISS), but not MDIS1, is involved in other physiological processes, such as stress responses upon cell wall damage, salt tolerance, root growth, and resistance to the important vascular fungal pathogen Fusarium oxysporum ${ }^{8-11}$. None of these processes are known to involve AtLURE1 peptides, and thus the identity and origin of the MIK2 ligand(s) remain unknown.

Here, we identify MIK2 as the receptor of the SCOOP family of phytocytokines. SCOOP12 binds directly to MIK2 to induce MIK2-BAK1 complex formation and activation of downstream signaling. All tested SCOOP peptides similarly induce MIK2dependent responses and MIK2-BAK1 complex formation. Moreover, we identify SCOOP-like sequences within Fusarium proteomes, whose corresponding synthetic peptides induce MIK2-dependent responses and MIK2-BAK1 association, suggesting MIK2 may directly perceive Fusarium.

\section{Results and discussion}

In an effort to understand how MIK2 regulates stress responses, we tested whether Arabidopsis thaliana (hereafter, Arabidopsis) mik2 mutant plants are affected in the production of reactive oxygen species (ROS) upon treatment with different immune elicitors. A clear defect in apoplastic ROS production was observed in mik2 plants for some, but not all elicitors tested based on elicitor origin (Supplementary Fig. 1). As a way to test the generality of these observations, other microbial and plant elicitors were tested, including the recently identified SERINE RICH ENDOGENOUS PEPTIDE 12 (SCOOP12) peptide ${ }^{12}$ that is proposed to function as a phytocytokine (a secreted peptide that regulates plant immune responses by analogy to cytokines in metazoans ${ }^{13}$ ). Surprisingly, unlike what was observed with flg22 or Pep1 (Supplementary Fig. 1), mik2 plants were insensitive to SCOOP12 treatment for all cellular responses measured (Fig. 1a-f and Supplementary Fig. 2a-d), i.e. ROS production increase in cytosolic $\mathrm{Ca}^{2+}$ concentration, mitogen-activated protein kinase phosphorylation, and seedling growth inhibition-all of which being hallmarks of immune signaling ${ }^{14}$. Importantly, the loss of MIK2 could be complemented with the expression of MIK2 under its native promoter (Supplementary Fig. 2e, f). That mik2 plants are blind to SCOOP12 suggests that MIK2 is the receptor for SCOOP12.
Plant cell-surface immune receptors (called pattern recognition receptors) are either RKs or receptor proteins (RPs), the latter lacking any obvious intracellular signaling domain ${ }^{15,16}$. The majority of known receptors for plant-derived or exogenous peptides are LRR-RKs or LRR-RPs that form ligand-induced complexes with LRR-RK co-receptors belonging to the SOMATIC EMBRYOGENESIS RECEPTOR KINASE (SERK) family ${ }^{1,17}$. Notably, SCOOP12-induced seedling growth inhibition was impaired in a bak1 (BRASSINOSTEROID INSENSITIVE 1-ASSOCIATED KINASE 1, BAK1 corresponds to SERK3) null mutant ${ }^{12}$. Similarly, we found that SCOOP12-induced ROS production is impaired in bak1-5 (Supplementary Fig. 3a, b), a bak1 allele that has a dominant-negative effect on other SERKs ${ }^{18-20}$. Furthermore, SCOOP12 treatment-induced BAK1 phosphorylation on serine 612 (Ser612) (Supplementary Fig. 3c)-a posttranslational modification required for immune signaling ${ }^{20}$. By comparison, SCOOP12-induced ROS production was not affected in sobir1-13 (Supplementary Fig. 3a, b), a null allele of the adapter LRR-RK SUPPRESSOR OF BAK1-INTERACTING RECEPTORLIKE KINASE 1 (SOBIR1) ${ }^{21}$, required by LRR-RPs to form active signaling complexes with SERK co-receptors ${ }^{15,16}$. Together, these data suggest that the SCOOP12 receptor is an LRR-RK. Consistently, we also found that optimal SCOOP12-induced ROS production occurs via components involved downstream of other LRR-RK-type PRRs ${ }^{22-25}$ (Supplementary Fig. 3d, e).

To further test if MIK2 is the SCOOP12 receptor, we transiently expressed MIK2 or a chimera between the MIK2 ectodomain and the intracellular domain of EF-TU RECEPTOR $(\mathrm{EFR})^{26}$ (MIK2-EFR) in Nicotiana benthamiana. This plant species is insensitive to SCOOP $12^{12}$ and its genome lacks any obvious MIK2 ortholog (Supplementary Fig. 4a-c). However, upon expression of MIK2 or MIK2-EFR, we could measure SCOOP12-induced ROS production in N. benthamiana, which was not observed when expressing a transformation control ( $\beta$-glucuronidase, GUS) or when expressing the reverse chimera EFR-MIK2 that is functional in elf18 perception (Fig. $1 \mathrm{~g}$, $\mathrm{h}$ and Supplementary Fig. $4 \mathrm{~d}-\mathrm{f}$ ). This data demonstrates that MIK2 is sufficient to confer SCOOP12 responsiveness, and indicates that the MIK2 ectodomain is involved in this recognition.

The current paradigm is that ligand-binding LRR-RKs undergo ligand-induced heterodimerization with a shape complementary co-receptor of the SERK family (or a related member of LRR-RK subfamily II) in order to activate signaling ${ }^{17,27,28}$. Having shown that SCOOP12-induced responses are BAK1-dependent (Supplementary Fig. 3a-c and ref. ${ }^{12}$ ), we tested whether SCOOP12 could induce MIK2-BAK1 complex formation. Stably expressed MIK2 fused to fluorescent epitope tags (MIK2-FP) coimmunoprecipitated endogenous BAK1 specifically upon treatment with SCOOP12 (Fig. 2a and Supplementary Fig. 5). In contrast, BAK1 co-immunoprecipitated with EFR-GFP upon elf18 but not SCOOP12 treatment in Arabidopsis (Fig. 2a). Consistent with this specific ligand-induced heteromerization, SCOOP12-induced BAK1-S612 phosphorylation was abolished in mik2 mutant plants (Fig. 2b). Finally, we tested if the MIK2 ectodomain (MIK2 ${ }^{\mathrm{ECD}}$ ) is sufficient for SCOOP12 binding in vitro. For this, we heterologously expressed $\mathrm{MIK} 2{ }^{\mathrm{ECD}}$ (residues 1-709) in insect cells, and performed binding assays using isothermal titration calorimetry. MIK2 ${ }^{\mathrm{ECD}}$ directly senses SCOOP12 with a binding affinity of $\sim 4.6 \mu \mathrm{M}$. We next quantified the affinity of the co-receptor to the MIK2-SCOOP12 complex. In the presence of SCOOP12, BAK1 strongly binds MIK2 with a dissociation constant in the mid-nanomolar range $(\sim 275 \mathrm{nM})$, which is comparable to its bioactivity (Fig. 2c, d and Supplementary Figs. 6, 7a). This suggests that SCOOP12 itself promotes receptorco-receptor association, as previously reported for other ligandLRR-RK pairs ${ }^{17,29}$. This result is in good agreement with the 
a

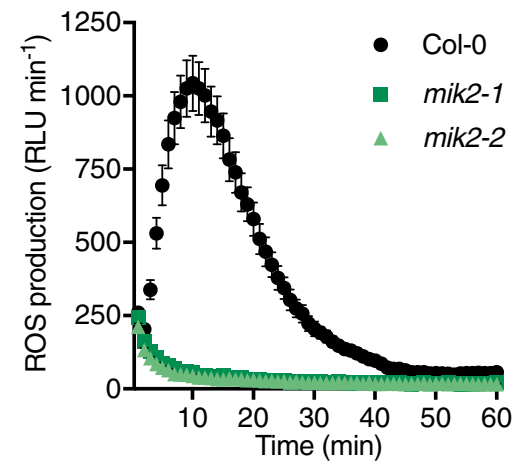

C

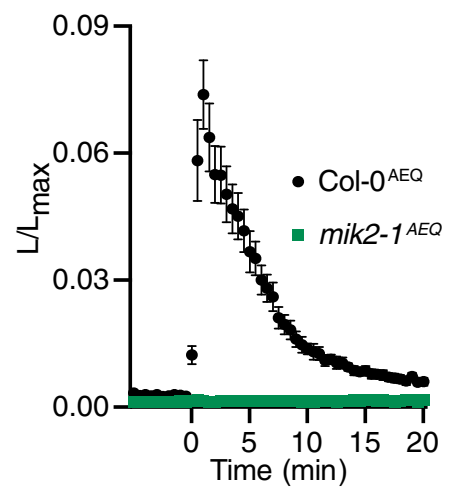

e
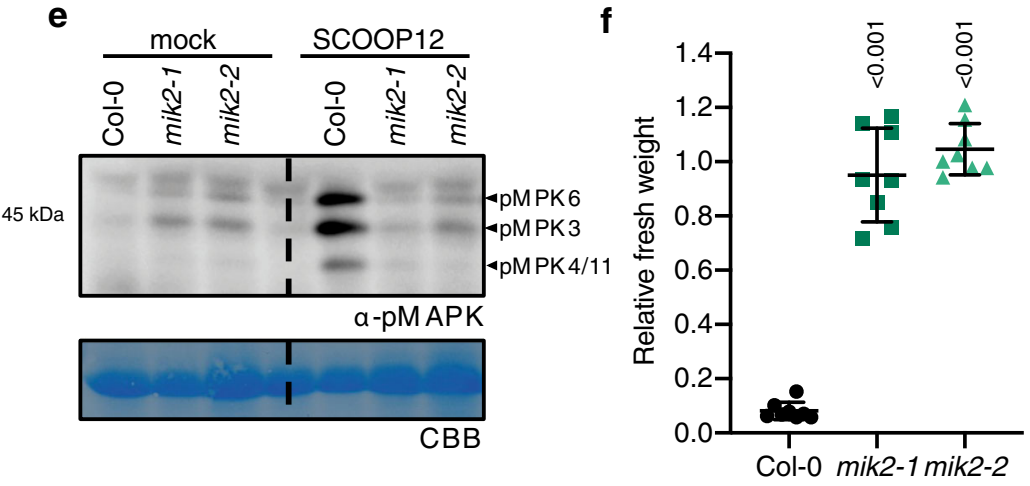

$\mathbf{g}$

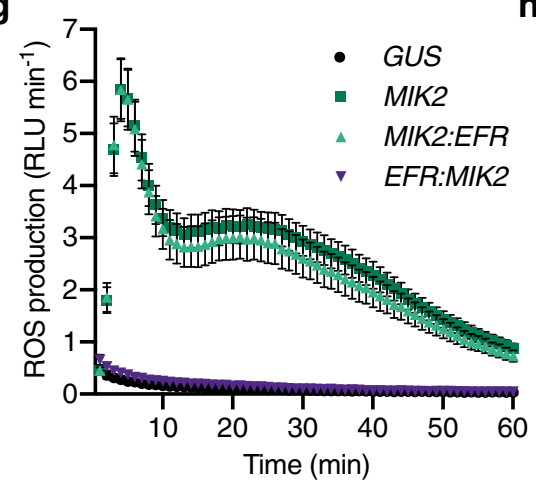

b

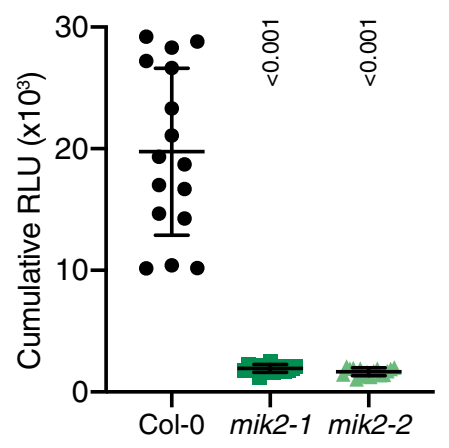

d



f

h

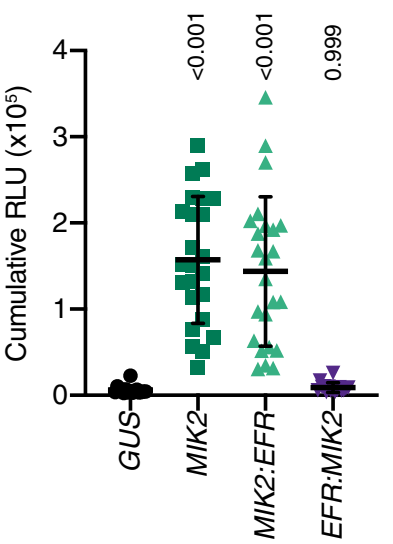

formation of a stable, SCOOP12-dependent MIK2-BAK1 complex in size-exclusion chromatography experiments (Fig. 2e). Together with the SERK-dependency of SCOOP12-induced responses (Supplementary Fig. $3 \mathrm{a}-\mathrm{c}$ and ref. ${ }^{12}$ ) and SCOOP12induced MIK2-BAK1/SERK association in planta (Fig. 2a and Supplementary Fig. 5), these in vitro data demonstrate that MIK2 is sufficient for SCOOP12 perception, and thus that MIK2 is the SCOOP12 receptor.

PROSCOOP12 is part of a 14-member family in Arabidopsis defined by the presence of a signal peptide, a pro-peptide region, a putative protease cleavage site, and a predicted mature peptide encompassing a serine- and glycine-rich 13-amino-acid epitope 
Fig. 1 MIK2 is required and sufficient for SCOOP12-induced responses. a, b ROS production in leaf disks collected from 4-week-old A. thaliana plants induced by $1 \mu \mathrm{M}$ SCOOP12 application ( $n=16$ leaf disks). a Points represent mean; error bars represent S.E.M. b Integrated ROS production over 40 min. Line represents mean; error bars represent S.D. c, d The mik2-1 mutation was crossed into the pUBQ10::AEQUORIN background, and calcium levels were measured in seedlings relative to maximum upon discharge following the addition of $100 \mathrm{nM} \mathrm{SCOOP12}$ ( $n=12$ seedlings). c Points represent mean; error bars represent S.E.M. d Maximum relative SCOOP12-induced calcium levels \pm S.D. e Western blot using $\alpha$-p42/p44-ERK recognizing phosphorylated MPK6, MPK3, and MPK4/11 in seedlings treated with $100 \mathrm{nM} \mathrm{SCOOP12}$ or mock for 15 min. Membranes were stained with CBB, as a loading control.

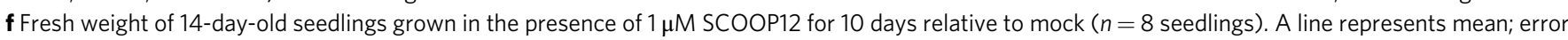
bars represent S.D. $\mathbf{g}, \mathbf{h}$ ) ROS production in leaf disks of $N$. benthamiana transiently expressing the defined constructs induced by $1 \mu \mathrm{M}$ SCOOP12 application $(n=24$ leaf disks). $\mathbf{g}$ Points represent mean; error bars represent S.E.M. $\mathbf{h}$ Integrated ROS production over 60 min. A line represents mean; error bars represent S.D. P-values indicate significance relative to the WT control (or GUS transformed control) in a Tukey's multiple comparisons test following oneway ANOVA. All experiments were repeated and analyzed three times with similar results. ROS reactive oxygen species, CBB Coomassie brilliant blue.

a

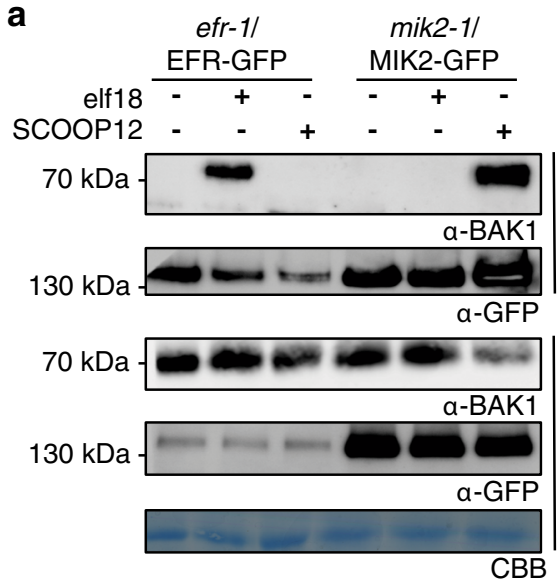

b

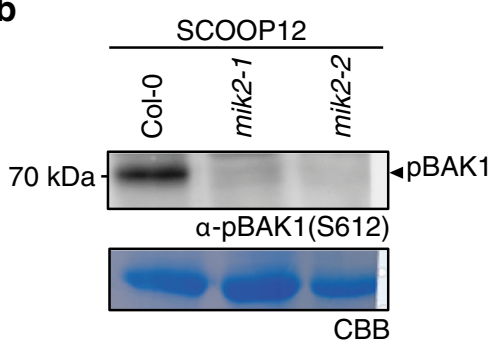

e
C

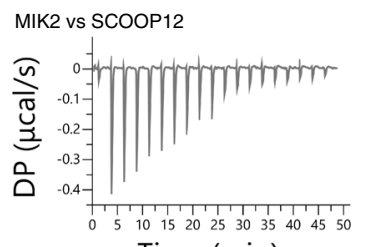

Time $(\mathrm{min})$

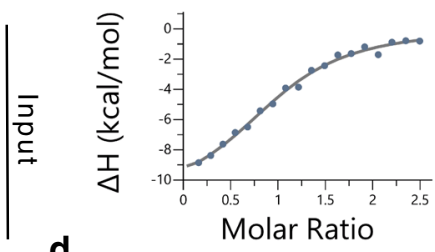

d

\begin{tabular}{llcrrr} 
Ligand (Syringe) & Protein (Cell) & $\mathrm{pH}$ & $\mathrm{Kd}(\mathrm{nM})$ & $\Delta \mathrm{H}(\mathrm{kcal} / \mathrm{mol})$ & $\mathrm{N}$ \\
\hline SCOOP12 & MIK2 & 5 & $4560 \pm 299$ & $-12.4 \pm 4.3$ & 1 \\
BAK1 & MIK2-SCOOP12 & 5 & $275 \pm 10$ & $-10.5 \pm 0.8$ & 1
\end{tabular}

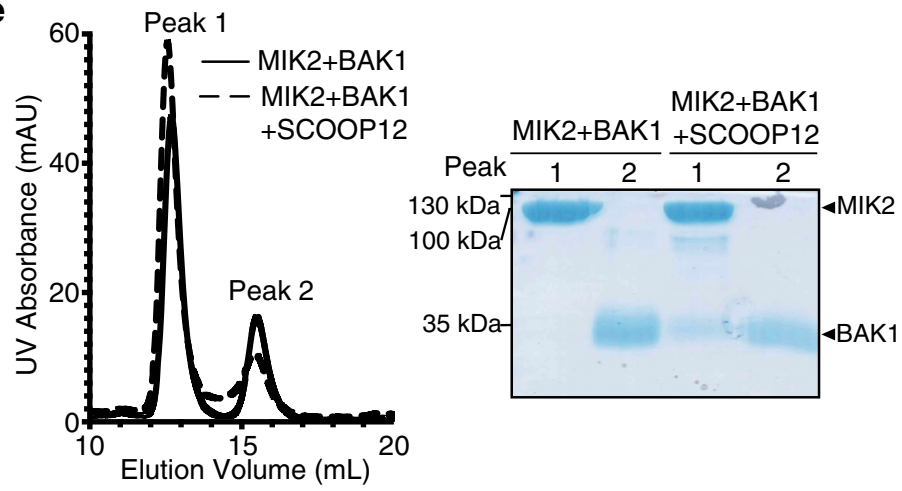

Fig. 2 SCOOP12 induces MIK2-BAK1 complex formation. a Co-immunoprecipitation of BAK1 with MIK2-GFP from mik2-1/MIK2-GFP and EFR-GFP from efr-1/EFR-GFP seedlings treated with $1 \mu \mathrm{M}$ elf18, $1 \mu \mathrm{M}$ SCOOP12, or water for $10 \mathrm{~min}$. Western blots were probed with antibodies $\alpha$-GFP and $\alpha$-BAK1. b Western blot using $\alpha$-pBAK1(Ser612) of seedlings after 15 min treatment with $100 \mathrm{nM} \mathrm{SCOOP12.} \mathrm{a,} \mathrm{b} \mathrm{were} \mathrm{repeated} \mathrm{three} \mathrm{times} \mathrm{with} \mathrm{similar} \mathrm{results.}$ c ITC experiments of MIK2 vs SCOOP12, and MIK2-SCOOP12 complex vs BAK1. Representative raw thermogram plots of ITC experiments. d ITC table summarizes of MIK2 vs SCOOP12, and MIK2-SCOOP12 vs BAK1. The binding affinities between MIK2 and SCOOP12, and MIK2-SCOOP12 and BAK1, are reported as $\mathrm{K}_{d}$ (dissociation constant, in nanomoles). The $N$ indicates the reaction stoichiometry $(N=1$ for a 1:1 interaction). $\Delta \mathrm{H}$ indicates the enthalpy variation. Values indicated in the table are means \pm SD of independent experiments $(n=3)$. Corresponding ITC runs are reported in Supplementary Figure 6. e Analytical SEC (left panel) of MIK2-BAK1 complex in the presence and absence of SCOOP12. An SDS-PAGE of the peak fractions is shown alongside (right panel). ITC isothermal titration calorimetry, SEC size-exclusion chromatography.

that was shown, at least for SCOOP12, to be biologically active in the nanomolar range when used as a synthetic peptide (Supplementary Fig. 7a, Supplementary Table 1, and ref. ${ }^{12}$ ). Importantly, deletion of the C-terminal arginine residue in SCOOP12 (SCOOP12 $\Delta$ ) abolished its activity (Supplementary Fig. 7b), suggesting that the predicted 13-mer corresponds to a minimal bioactive SCOOP epitope. Notably, while MIK2 is the SCOOP12 receptor, a proscoop12 loss-of-function mutant ${ }^{12}$ did not phenocopy mik2, as tested for ROS production and root skewing (Supplementary Fig. 8), suggesting that additional SCOOPs are perceived by MIK2.

A multiple alignment of Arabidopsis SCOOP sequences corresponding to the defined bioactive epitope revealed poor conservation beyond two conserved serines at positions 5 and 7 
(previously shown to be essential for SCOOP12 activity ${ }^{12}$ ), and the presence of a glycine-rich C-terminal region for SCOOP family members (Fig. 3a). Interestingly, the C-terminal sequence of SCOOP peptides differs from that of other known serine/ glycine/proline-rich plant peptides (e.g. CLE, Pep, IDA, PIP, or CEP families ${ }^{2}$ ), which often contain an arginine-histidineasparagine motif ${ }^{17}$ suggesting a potential different mode of ligand-binding-something which will need to be tested in the future in structural studies.

We synthesized peptides corresponding to all Arabidopsis SCOOPs with available sequence information on TAIR10 (Supplementary Table 1), and found that all but three-SCOOP6, 8, and 11 -were active in at least one assay tested at either $1 \mu \mathrm{M}$ or $10 \mu \mathrm{M}$ (Fig. 3b, c and Supplementary Fig. 9a, b). Of note, in the a

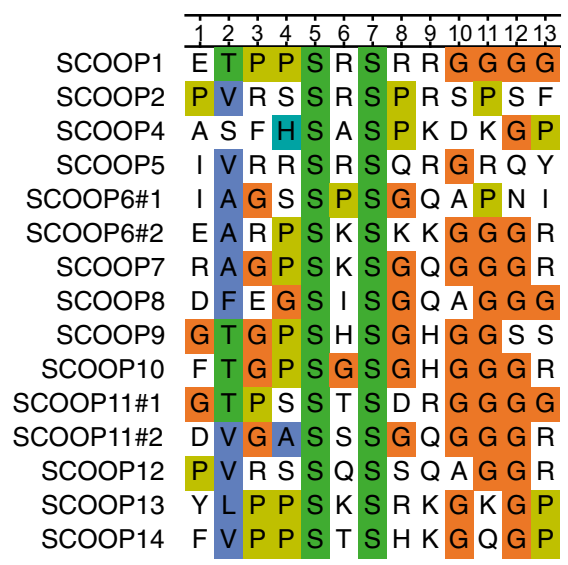

b

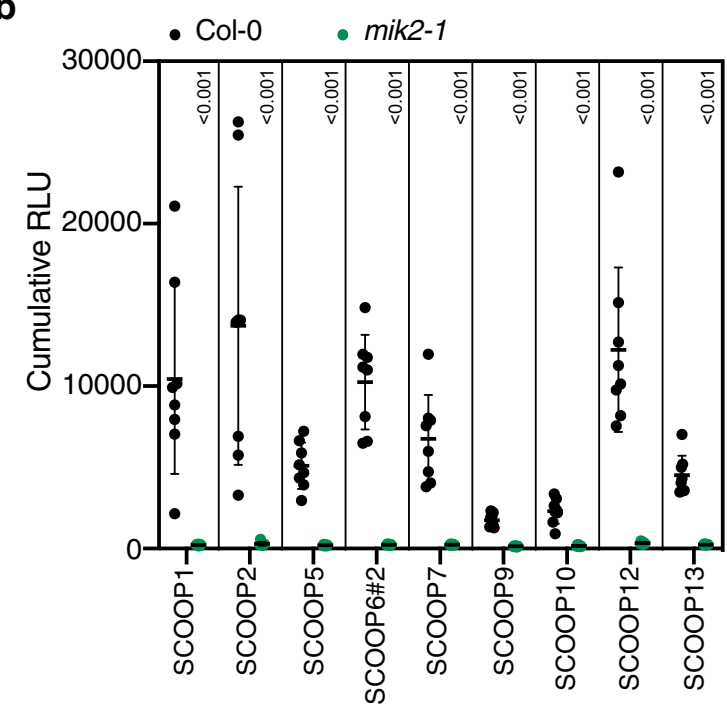

C

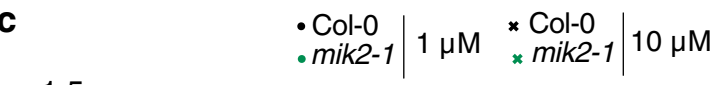

b

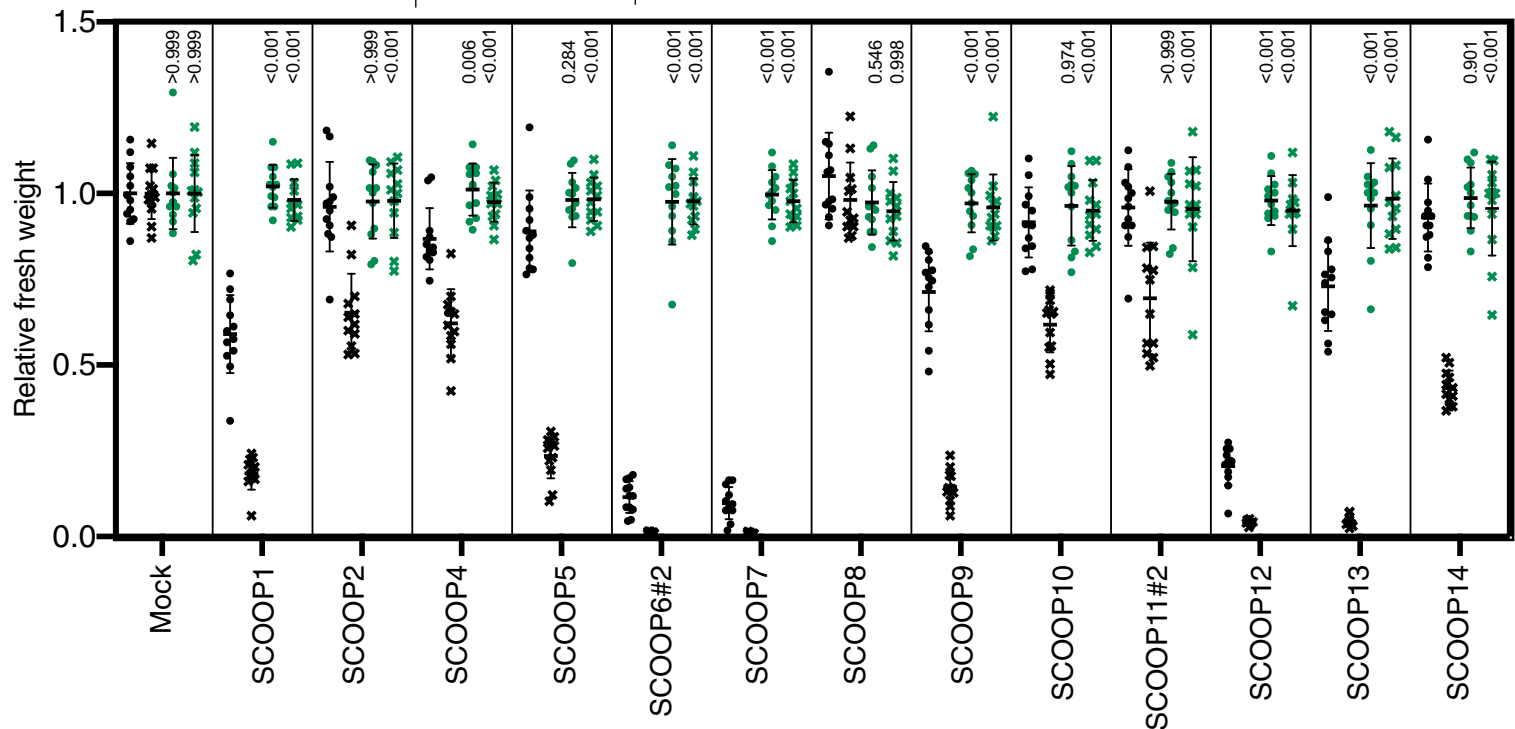

d

mik2-1/MIK2-GFP

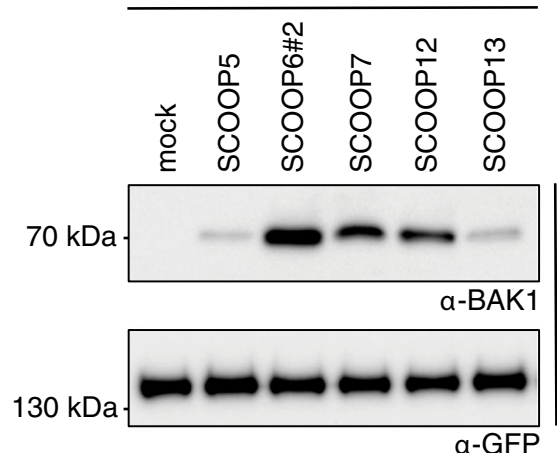

mik2-1/MIK2-GFP

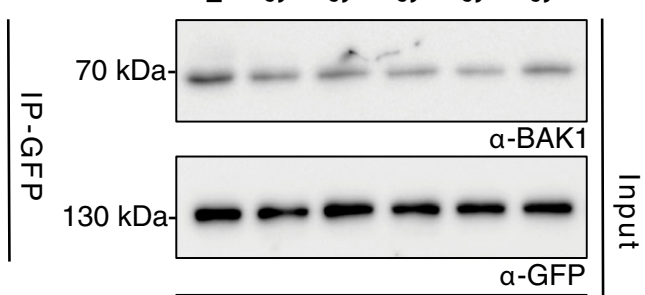

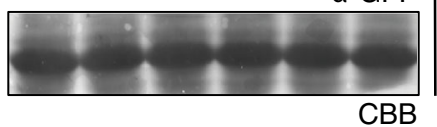


Fig. 3 Divergent SCOOP peptides induce MIK2-dependent responses. a Alignment of SCOOP motifs from A. thaliana. $\mathbf{b}$ ROS production in leaf disks collected from 4-week-old $A$. thaliana plants induced by application of $1 \mu \mathrm{M}$ SCOOP peptides in Col-0 (black) or mik2-1 (green) ( $n=8$ leaf disks). A line represents mean; error bars represent S.D. P-values indicate significance relative to the WT control as indicated by two-tailed $T$ test $\mathbf{c}$ Fresh weight of 14-day-old seedlings grown in the presence of $1 \mu \mathrm{M} \mathrm{SCOOP} \mathrm{peptides} \mathrm{for} 10$ days relative to mock. A line represents mean; error bars represent S.D. $(n=$ 12 seedlings). A two-way ANOVA indicated a significant effect of treatment and genotype $(P<0.0001) ; P$-values indicate significance relative to Col-0 in a Sidak's multiple comparison test between genotypes within each treatment. d Co-immunoprecipitation of BAK1 with MIK2-GFP from mik2-1/MIK2-GFP seedlings treated with $1 \mu \mathrm{M}$ SCOOP peptides, or water for $10 \mathrm{~min}$. Western blots were probed with antibodies $\alpha$-GFP and $\alpha$-BAK1. These experiments were performed and analyzed three times with similar results. These experiments were performed and analyzed three times with similar results. ROS reactive oxygen species.

absence of current knowledge about the physiological concentration of SCOOP peptides released in the plant apoplast, these arbitrary concentrations were simply chosen to test for the ability of the peptides to induce the responses measured. Notably, upon careful examination of PROSCOOP6 and PROSCOOP11, we noticed additional SCOOP motifs in these proteins ${ }^{12}$ - which we named SCOOP6 $\sharp 2$ and SCOOP $11 \sharp 2$ (in contrast to SCOOP6 $\sharp 1$ and SCOOP11 $\sharp 1$ previously tested). Synthetic peptides derived from these alternative SCOOP motifs turned out to be active in multiple assays (Supplementary Fig. 9c-e). Having identified twelve bioactive SCOOP peptides, we then tested if their activities were also MIK2-dependent. Using ROS production and seedling growth inhibition as a readout, we found that, despite the low level of sequence similarity, all active SCOOP peptides required MIK2 (Fig. 3b, c). Furthermore, ligand-induced MIK2-BAK1 association was also observed in response to selected SCOOPs (Fig. 3d). Together, these experiments indicate that MIK2 is the receptor for the divergent family of SCOOP peptides.

The finding that a single receptor is sufficient and necessary for the perception of all members of a given (divergent) peptide family is so far unique to the SCOOP-MIK2 ligand-receptor system. Indeed, members of other plant peptides families (e.g. CLEs, RALFs) are often distinctively or conjunctively perceived by several related receptors ${ }^{30,31}$, or are perceived by partially redundant-related receptors with often distinct quantitative contributions (e.g. perception of CIFs, IDLs, and AtPeps) $2,32,33$.

MIK2 is required for resistance to F. oxysporum ${ }^{9}$ and is required for immune responses to a Fusarium extract ${ }^{11}$. One hypothesis would thus be that perception of the Fusarium extract leads to SCOOP perception by MIK2, potentially via the control of PROSCOOP expression, PROSCOOP cleavage, and/or SCOOP secretion. While theoretically possible, this would however not be in line with the responses to the Fusarium extract that can be measured within minutes ${ }^{11}$. Homologous sequences to plant peptides have been reported in plant-associated organisms, whose recognition and bioactivity depend on the corresponding plant receptors $^{34-42}$. Using BLAST and MAST algorithms, we identified several SCOOP-like motifs from Fusarium proteomes and ordered the corresponding synthetic peptides (Fig. 4a, Supplementary Fig. 10, and Supplementary Table 2). Two of these peptides, derived from the proteins A0A0M9EVJ7 and A0A0D2XZ19, were able to induce immune responses in a MIK2-dependent manner (Fig. 4a-e and Supplementary Fig. 11a-e). Response to these peptides was also BAK1-dependent, and peptide treatmentinduced MIK2-BAK1 association (Fig. 4b-f and Supplementary Fig. $11 b, c)$, demonstrating that these synthetic peptides are sufficient as ligands for the MIK2-BAK1 complex. Notably, whilst the SCOOP-like motif present in A0A0M9EVJ7 is relatively poorly conserved, the A0A0D2XZ19 motif shows conservation within related fungal proteomes (Fig. 4a and Supplementary Fig. 11f), consistent with the presence of a MIK2-dependent eliciting activity in extracts from different Fusarium species ${ }^{11}$. Together, these results indicate that the elicitor present in Fusarium extracts may derive from Fusarium proteins harboring a SCOOP-like motif recognized by MIK2. It however remains to be established in future studies whether these epitope(s) facilitate non-self recognition in the context of an infection, thus contributing to the enhanced susceptibility of mik2 mutants to Fusarium ${ }^{9,11}$.

In conclusion, the characterization of the SCOOP-MIK2 ligand-receptor module represents a significant advance in our understanding of the mechanism through which phytocytokines are perceived by plant cells. Given the multiple physiological functions of MIK2, the identification of SCOOPs as MIK2 ligands will enable the future dissection of the roles of these divergent peptides in diverse pathways and on the molecular basis of their perception.

\section{Methods}

No statistical methods were used to predetermine the sample size. The experiments were not randomized, and investigators were not blinded to allocation during experiments and outcome assessment.

Plant material and growth conditions. Arabidopsis thaliana ecotype Columbia (Col-0) and Wassilewskija (Ws-2) were used as wild-type controls. Plants for ROS burst assays were grown in individual pots at $21^{\circ} \mathrm{C}$ with a $10-\mathrm{h}$ photoperiod. Seeds grown on plates were surface sterilized using chlorine gas for $5-6 \mathrm{~h}$, and sown on Murashige and Skoog (MS) media supplemented with vitamins, $1 \%$ sucrose, and $0.8 \%$ agar and stratified at $4{ }^{\circ} \mathrm{C}$ for $2-3$ days. Nicotiana benthamiana plants were grown on peat-based media at $24^{\circ} \mathrm{C}$, with 16 -h photoperiod.

The following $A$. thaliana mutants in Col-0 background (except otherwise indicated) were used: mik2-1 (SALK_061769) ${ }^{3}$ (kindly provided by Wei-Cai Yang), mik2-2 (SALK_046987) ${ }^{9}$, mik2-4 (FLAG_518G04), bak1-5 ${ }^{18}$, sobir1-13

(SALK_009453) ${ }^{21}$ (kindly provided by Yuelin Zhang), cpk28-1 (GK-523B08) ${ }^{25}$, rbohD ${ }^{23}, c p k 5 c p k 6 c p k 11^{22}$ (kindly provided by Jen Sheen), bik1 pbl1 ${ }^{43}$ (kindly provided by Jian-Min Zhou), pro35S::GFP-LTI6B ${ }^{44}$, and proscoop $12^{12}$ (kindly provided by Jean-Pierre Renou). The transgenic lines efr/EFR-GFP and proUBQ10:: $A E Q$ (kindly provided by Justin Lee) lines were described ${ }^{45,46}$. The mik2-1/pro35S: MIK2-GFP line was generated in this study. mik2-1 plants were transformed with the MIK2 coding sequence in the pEARLEYGATE103 binary vector using floral dip transformation ${ }^{47}$. Transformants were selected to homozygosity using phosphinotricin.

Synthetic peptides. All synthetic peptides were ordered at $>80 \%$ purity (physiological assays), $>95 \%$ purity (biochemical assays) (EZBiolabs). Sequences of all peptides can be found in Supplementary Table 1. The gene models from which the peptide sequences were extracted are listed in Supplementary Table 4.

Molecular cloning. For overexpression of MIK2 in A. thaliana and N. benthamiana, the MIK2.1 coding sequence was PCR amplified from Arabidopsis Col-0 cDNA using gene-specific primers (Supplementary Table 3 ) and was cloned into pENTR using the D-TOPO kit (Invitrogen) subsequently recombined using LR Clonase II (Invitrogen) into the pEarleygate103 expression vector downstream of the $35 \mathrm{~S}$ promoter and in frame with a C-terminal poly-His-GFP tag ${ }^{48}$. Generation of the chimeric receptors was performed using overlap extension PCR from EFR and MIK2 entry clones. The final PCR product was then recombined into the pEarleygate103 expression vector using LR Clonase II (Invitrogen). Native promoter sequences were amplified and cloned with C-terminal tags into pICSL86955 using BsaI restriction sites. All clones were verified by Sanger sequencing.

ROS measurement. Leaf disks were harvested from 4-week-old Arabidopsis plants or 3-week-old $N$. benthamiana using a 4 -mm diameter biopsy punch (Integra ${ }^{\text {Tx }}$ Miltex $^{\text {тM }}$. Leaf disks were floated overnight on $100 \mu \mathrm{L}$ of distilled water in white 96-well-plates (Greiner Bio-One). Prior to ROS measurement, the water was removed and replaced with ROS assay solution (100 $\mu \mathrm{M}$ Luminol (Merck), 
Arabidopsis thaliana I SCOOP10

Fusarium langsethiae | A0A0M9EVJ7

Fusarium oxysporum (strain Fo5176) I F9FBF1

Fusarium oxysporum f. sp. cubense I N1RC68

Trichoderma atroviridis I G9P528

Arabidopsis thaliana I SCOOP12

Fusarium oxysporum f. sp. lycopersici I A0A0D2XZ19

Fusarium oxysporum (strain Fo5176) I F9F6H0

Fusarium oxysporum f. sp. cubense I N1RXB9

Trichoderma atroviridis I G9NTF3

Verticillium dahliae I A0A2J8DS38

Magnaporthe oryzae I G4MSI6

Neurospora crassa IQ7SAG1

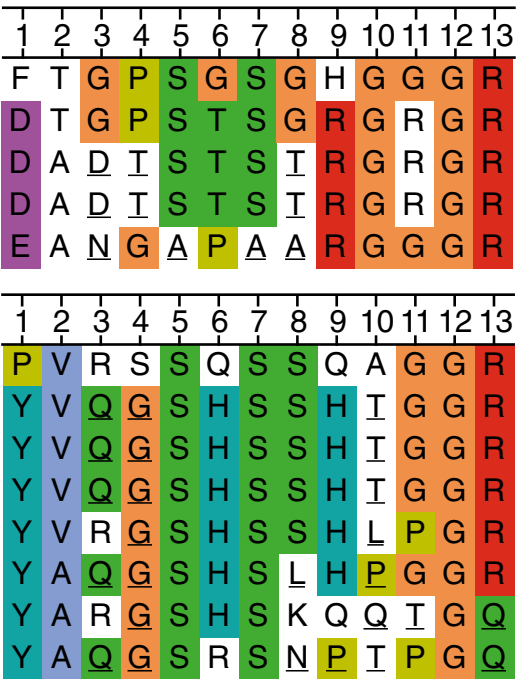

C

\section{b}

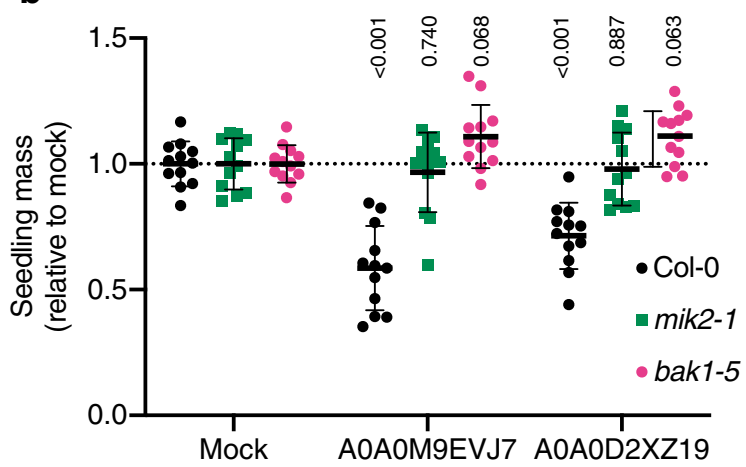

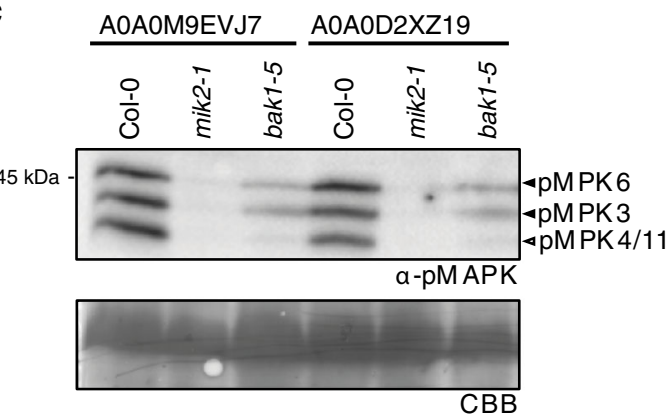
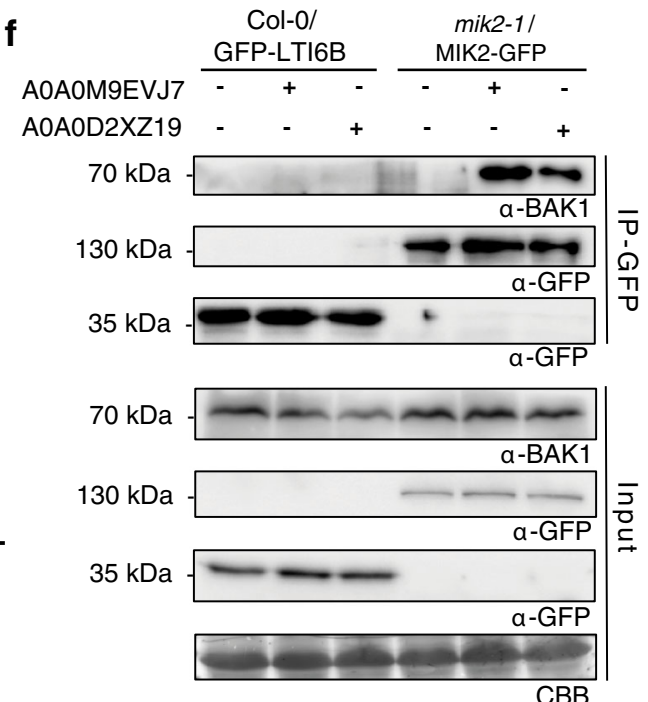

Fig. 4 Fusarium-derived peptides are induced MIK2-dependent responses. a Alignments of the SCOOP-like motifs from AOAOM9EVJ7 and AOAOD2XZ191 in closely related fungal species with the most closely related Arabidopsis SCOOP based on amino acid identity. Residues that are not found in any Arabidopsis SCOOP are underlined $\mathbf{b}$ Relative fresh weight of 14-day-old seedlings grown in the presence of $1 \mu$ M AOAOM9EVJ7 or A0A0D2XZ19 for 10 days relative to mock $(n=12$ seedlings). A line represents mean; error bars represent S.D. P-values are derived from a Dunnett's multiple comparison test comparing the treatment to mock for each genotype following a two-way ANOVA. c Western blot using $\alpha$-p42/p44-ERK recognizing phosphorylated MPK6, MPK3, and MPK4/11 in seedlings treated with $1 \mu$ M AOAOM9EVJ7, AOAOD2XZ19 or mock for 15 min. Membranes were stained with CBB, as a loading control. d, e Integrated ROS production in leaf disks collected from 4-week-old A. thaliana plants induced by d $1 \mu \mathrm{M}$ AOAOM9EVJ7 or e $1 \mu \mathrm{M}$ AOA0D2XZ19 application over $40 \mathrm{~min}(n=8$ leaf disks). Line represents mean; error bars represent S.D. P-values are derived from a Dunnett's multiple comparison test comparing the treatment to mock for each genotype following a one-way ANOVA. $\mathbf{f}$ Co-immunoprecipitation of BAK1 with MIK2-GFP from mik2-1/MIK2-GFP and GFP-LTI6B from Col-0/GFP-LTI6B seedlings treated with $1 \mu \mathrm{M}$ AOAOM9EVJ7, $1 \mu \mathrm{M}$ AOAOD2XZ19, or water for 10 min. Western blots were probed with antibodies $\alpha$-GFP and $\alpha$-BAK1. All experiments were performed and analyzed three times with similar results. CBB Coomassie brilliant blue, ROS reactive oxygen species. 
$20 \mu \mathrm{g} \mathrm{mL}^{-1}$ horseradish peroxidase (Merck)) with or without the addition of elicitors. Immediately following the addition of the assay solution light emission was measured from the plate using a HIGH-RESOLUTION PHOTON COUNTING SYSTEM (HRPCS218, Photek) equipped with a $20 \mathrm{~mm}$ F1.8 EX DG ASPHERICAL RF WIDE LENS (Sigma Corp).

Cytoplasmic calcium measurement. Arabidopsis seedlings were grown in 96-well plates (Greiner Bio-One) in $100 \mu \mathrm{L}$ liquid MS for 5 days. The evening before calcium measurements the liquid MS was replaced with $100 \mu \mathrm{L} 20 \mu \mathrm{M}$ coelenterazine (Merck) and the seedlings incubated in the dark overnight. The following morning the coelenterazine solution was replaced with $100 \mu \mathrm{L}$ water and rested for a minimum of $30 \mathrm{~min}$ in the dark. Readings were taken in a VARIOSKAN ${ }^{\mathrm{TM}}$ MUTIPLATE READER (ThermoFisher) using the injector to add $50 \mu \mathrm{L}$ of $3 \times$ concentrated elicitor solution or mock. $\mathrm{L}_{\max }$ was determined as the maximum luminescence emission upon discharge with $\left(1 \mathrm{M} \mathrm{CaCl}_{2}, 10 \%\right.$ ethanol).

Seedling growth inhibition. Four-day-old seedlings growing on MS plates were transferred individually into separate wells of transparent 48 -well tissue culture plates (Greiner Bio-One) containing $500 \mu \mathrm{L}$ of liquid MS media with/without elicitor addition. The plates were then transferred back to the growth conditions for an additional 10 days before seedlings were blot-dried and weighed.

Protein extraction and western blot. Two-week-old seedlings grown in liquid MS media (or N. benthamiana leaf tissue) were flash-frozen in liquid nitrogen. Plant tissue was ground in liquid nitrogen prior to boiling in $2 \times$ Laemmli sample buffer (4\% SDS, $20 \%$ glycerol, $10 \%$ 2-mercaptoethanol, $0.004 \%$ bromophenol blue, and $0.125 \mathrm{M}$ Tris-HCl; $\left(10 \mu \mathrm{L} \cdot \mathrm{mg}^{-1}\right.$ tissue) $)$ for $10 \mathrm{~min}$ at $95^{\circ} \mathrm{C}$. The samples were then spun at $13,000 \times g$ for 5 min prior to loading and running on SDS-PAGE gels of an appropriate concentration. Proteins were transferred onto PVDF membrane (ThermoFisher) prior to incubation with appropriate antibodies ( $\alpha$-pBAK1 $(\operatorname{Ser612})^{20}$ (1:2000); $\alpha$-GFP-HRP (sc-9996, Santa Cruz; 1:5000); $\alpha$-pMAPK (p44/42 MAPK (Erk1/2) antibody \#9102; 1:4000); $\alpha$-rabbit-HRP (A-0545, Merck; 1:10,000). Western blots were imaged with a LAS 4000 IMAGEQUANT SYSTEM (GE Healthcare). Staining of the blotted membrane with Coomassie brilliant blue was used to confirm loading.

Co-immunoprecipitation. Fifteen to twenty seedlings were grown in wells of a 6-well plate for 2 weeks in liquid MS media with gentle agitation (6 wells were used per treatment). The MS media was replaced the night before treatment. Seedlings were treated with $1 \mu \mathrm{M}$ elf18/SCOOP12 for $10 \mathrm{~min}$ before flash-freezing. Tissue was ground and proteins extracted in 1:1 $(\mathrm{v} / \mathrm{v})$ powdered tissue:extraction buffer (50 mM Tris-HCl pH 7.5, $150 \mathrm{mM} \mathrm{NaCl}, 10 \%$ glycerol, $5 \mathrm{mM}$ dithiothreitol, $1 \%$ protease inhibitor cocktail (P9599, Sigma Aldrich), $2 \mathrm{mM} \mathrm{Na}_{2} \mathrm{MoO}_{4}, 2.5 \mathrm{mM} \mathrm{NaF}$, $1.5 \mathrm{mM}$ activated $\mathrm{Na}_{3} \mathrm{VO}_{4}$ and $1 \%$ IGEPAL) for $30 \mathrm{~min}$ at $4{ }^{\circ} \mathrm{C}$. Cell debris was removed via centrifugation. For immunoprecipitation, GFP-TRAP/RFP-TRAP AGAROSE BEADS (ChromoTek) were incubated with extracts for $3 \mathrm{~h}$ at $4{ }^{\circ} \mathrm{C}$ and washed three times in wash buffer $(50 \mathrm{mM}$ Tris- $\mathrm{HCl} \mathrm{pH} 7.5,150 \mathrm{mM} \mathrm{NaCl}, 10 \%$ glycerol, $5 \mathrm{mM}$ dithiothreitol, $1 \%$ protease inhibitor cocktail (P9599, Sigma Aldrich), $2 \mathrm{mM} \mathrm{Na} 2 \mathrm{MoO} 4,2.5 \mathrm{mM} \mathrm{NaF}, 1.5 \mathrm{mM}$ activated $\mathrm{Na}_{3} \mathrm{VO}_{4}$, and $0.1 \%$ IGEPAL) before adding $2 \times$ Laemmli sample buffer and incubating for $10 \mathrm{~min}$ at $95^{\circ} \mathrm{C}$. Detection was carried out by SDS-PAGE and western blots using $\alpha$-BAK $1{ }^{19}$ $\alpha$-RFP (ab34771, Abcam) and a-GFP-HRP (sc-9996, Santa Cruz) antibodies. $\alpha$-rabbit-HRP (A-0545, Merck) was used to detect $\alpha$-BAK1 and $\alpha$-RFP.

Transient expression in Nicotiana benthamiana. Agrobacterium tumefaciens strain GV3101 transformed with the corresponding construct were grown overnight in L-media and spun-down. For ROS assays the bacteria were resuspended in $10 \mathrm{mM} \mathrm{MgCl}_{2}$ and adjusted to O.D.600 $=0.2$ prior to infiltration into the youngest fully expanded leaves of 3-week-old plants. Leaf disks were collected $24 \mathrm{~h}$ later, and ROS assays were performed as described for Arabidopsis.

Root skewing. Seeds were sown directly on MS agar square plates and stratified for 2 days at $4{ }^{\circ} \mathrm{C}$. Plates were transferred to $22^{\circ} \mathrm{C}$ under a 16 -h photoperiod, in an upright position for 9 days. The root angle was measured by the ImageJ software, as performed previously'.

Protein expression and purification. Codon-optimized synthetic genes for expression in Spodoptera frugiperda (Invitrogen GeneArt), coding for Arabidopsis thaliana MIK2 (residues 1-709) and BAK1 (1-220) ectodomains were cloned into a modified pFastBac (Geneva Biotech) vector, providing a TEV (tobacco etch virus protease) cleavable C-terminal StrepII-9xHis tag. For protein expression, Trichoplusia $n i$ Tnao 38 cells $^{49}$ were infected with MIK2 or BAK1 virus with a multiplicity of infection (MOI) of 3 and incubated for $1 \mathrm{~d}$ at $28^{\circ} \mathrm{C}$ and $2 \mathrm{~d}$ at $22^{\circ} \mathrm{C}$ at $110 \mathrm{rpm}$. The secreted proteins were purified from the supernatant by sequential $\mathrm{Ni}^{2+}$ (HisTrap Excel column; GE Healthcare; equilibrated in $25 \mathrm{mM} \mathrm{KP}_{\mathrm{i}} \mathrm{pH} 7.8$, $500 \mathrm{mM} \mathrm{NaCl}$ ) and StrepII (Strep-Tactin Superflow high capacity (IBA Lifesciences) equilibrated in $25 \mathrm{mM}$ Tris $\mathrm{pH} 8.0,250 \mathrm{mM} \mathrm{NaCl}, 1 \mathrm{mM}$ EDTA) affinity chromatography. All proteins were incubated with TEV protease to remove the tags. Proteins were further purified by SEC on a Superdex 200 increase 10/300 GL column (GE Healthcare), equilibrated in $20 \mathrm{mM}$ sodium citrate $\mathrm{pH} 5.0,150 \mathrm{mM}$ $\mathrm{NaCl}$. For biochemical experiments, proteins were concentrated using Amicon Ultra concentrators (Millipore, molecular weight cutoff 10,000 and 30,000).

ITC experiments. Experiments were performed at $25^{\circ} \mathrm{C}$ using a MicroCal PEAQ ITC (Malvern Instruments) with a $200-\mu \mathrm{L}$ standard cell and a $40-\mu \mathrm{L}$ titration syringe. MIK2 and BAK1 ectodomains were gel filtrated into $\mathrm{pH} 5$ ITC buffer ( $20 \mathrm{mM}$ sodium citrate $\mathrm{pH} 5.0,150 \mathrm{mM} \mathrm{NaCl}$ ). SCOOP12 peptide powder was dissolved in the same buffer to obtain the desired concentration. A typical experiment consisted of injecting $1 \mu \mathrm{L}$ of a $200 \mu \mathrm{M}$ solution of the ligand into $20 \mu \mathrm{M}$ MIK2 solution in the cell at $150 \mathrm{~s}$ intervals. BAK1 vs MIK2-SCOOP12 experiments were performed by titrating $200 \mu \mathrm{M}$ BAK1 onto the MIK2-SCOOP12 complex in the cell, using the same injection pattern. ITC data were corrected for the heat of dilution by subtracting the mixing enthalpies for titrant solution injections into protein-free ITC buffer. Experiments were done in triplicates and data were analyzed using the MicroCal PEAQ-ITC Analysis Software provided by the manufacturer. All ITC runs used for data analysis had an $N$ ranging between 0.8 and 1.3. The $N$ values were fitted to 1 in the analysis.

Similarity/identity matrices. SIAS was used to generate similarity and identity matrices (http://imed.med.ucm.es/Tools/sias.html).

Species divergence estimations. The timing of fungal species divergence was estimated using TimeTree ${ }^{50-53}$.

Statistical analysis. Statistical analysis was performed in GraphPad Prism 7.0. (GraphPad Software, http://www.graphpad.com) unless stated otherwise. Dot plots were used to show individual data points wherever possible. $P$-values $<0.05$ were considered nonsignificant. Sample sizes, statistical tests used, and $P$-values are stated in the figure legends.

Reporting summary. Further information on research design is available in the Nature Research Reporting Summary linked to this article.

\section{Data availability}

For blot source images, see Supplementary Fig. 1. All other data or materials can be obtained from the corresponding author upon request. Source data are provided with this paper.

Received: 28 October 2020; Accepted: 23 December 2020; Published online: 29 January 2021

\section{References}

1. Dievart, A., Gottin, C., Périn, C., Ranwez, V. \& Chantret, N. Origin and diversity of plant receptor-like kinases. Annu. Rev. Plant Biol. 71, 131-156 (2020).

2. Olsson, V. et al. Look closely, the beautiful may be small: precursor-derived peptides in plants. Annu. Rev. Plant Biol. 70, 153-186 (2019).

3. Wang, T. et al. A receptor heteromer mediates the male perception of female attractants in plants. Nature 531, 241-244 (2016).

4. Cheung, A. Y. \& Wu, H. M. Plant biology: LURE is bait for multiple receptors Nature 531, 178-180 (2016).

5. Takeuchi, H. \& Higashiyama, T. Tip-localized receptors control pollen tube growth and LURE sensing in Arabidopsis. Nature 531, 245-248 (2016)

6. Zhang, X. et al. Structural basis for receptor recognition of pollen tube attraction peptides. Nat. Commun. 8, 1331 (2017).

7. Zhong, S. et al. Cysteine-rich peptides promote interspecific genetic isolation in Arabidopsis. Science 364, eaau9564 (2019).

8. Julkowska, M. M. et al. Natural variation in rosette size under salt stress conditions corresponds to developmental differences between Arabidopsis accessions and allelic variation in the LRR-KISS gene. J. Exp. Bot. 67, 2127-2138 (2016).

9. Van der Does, D. et al. The Arabidopsis leucine-rich repeat receptor kinase MIK2/LRR-KISS connects cell wall integrity sensing, root growth and response to abiotic and biotic stresses. PLoS Genet. 13, e1006832 (2017).

10. Engelsdorf, T. et al. The plant cell wall integrity maintenance and immune signaling systems cooperate to control stress responses in Arabidopsis thaliana. Sci. Signal 11, eaao3070 (2018). 
11. Coleman, A. D. et al. The Arabidopsis leucine-rich repeat receptor-like kinase MIK2 is a crucial component of early immune responses to a fungal-derived elicitor. New Phytol. https://doi.org/10.1111/NPH.17122 (2020).

12. Gully, K. et al. The SCOOP12 peptide regulates defense response and root elongation in Arabidopsis thaliana. J. Exp. Bot. 70, 1349-1365 (2019).

13. Gust, A. A., Pruitt, R. \& Nürnberger, T. Sensing danger: key to activating plant immunity. Trends Plant Sci. 22, 779-791 (2017).

14. Yu, X., Feng, B., He, P. \& Shan, L. From chaos to harmony: responses and signaling upon microbial pattern recognition. Annu. Rev. Phytopathol. 55, 109-137 (2017).

15. Boutrot, F. \& Zipfel, C. Function, discovery, and exploitation of plant pattern recognition receptors for broad-spectrum disease resistance. Annu. Rev. Phytopathol. 55, 257-286 (2017).

16. Tang, D., Wang, G. \& Zhou, J. M. Receptor kinases in plant-pathogen interactions: more than pattern recognition. Plant Cell 29, 618-637 (2017).

17. Hohmann, U., Lau, K. \& Hothorn, M. The structural basis of ligand perception and signal activation by receptor kinases. Annu. Rev. Plant Biol. 68, 109-137 (2017).

18. Schwessinger, B. et al. Phosphorylation-dependent differential regulation of plant growth, cell death, and innate immunity by the regulatory receptor-like kinase BAK1. PLoS Genet. 7, e1002046 (2011).

19. Roux, M. et al. The Arabidopsis leucine-rich repeat receptor-like kinases BAK1/SERK3 and BKK1/SERK4 are required for innate immunity to hemibiotrophic and biotrophic pathogens. Plant Cell 23, 2440-2455 (2011).

20. Perraki, A. et al. Phosphocode-dependent functional dichotomy of a common co-receptor in plant signalling. Nature 561, 248-252 (2018).

21. Gao, M. et al. Regulation of cell death and innate immunity by two receptorlike kinases in Arabidopsis. Cell Host Microbe 6, 34-44 (2009).

22. Boudsocq, $M$. et al. Differential innate immune signalling via $\mathrm{Ca}(2+)$ sensor protein kinases. Nature 464, 418-422 (2010).

23. Kadota, Y. et al. Direct regulation of the NADPH oxidase RBOHD by the PRR-associated kinase BIK1 during plant immunity. Mol. Cell. 54, 43-55 (2014).

24. Li, L. et al. The FLS2-associated kinase BIK1 directly phosphorylates the NADPH oxidase RbohD to control plant immunity. Cell Host Microbe 15, 329-338 (2014).

25. Monaghan, J. et al. The calcium-dependent protein kinase CPK28 buffers plant immunity and regulates BIK1 turnover. Cell Host Microbe 16, 605-615 (2014).

26. Zipfel, C. et al. Perception of the bacterial PAMP EF-Tu by the receptor EFR restricts Agrobacterium-mediated transformation. Cell 125, 749-760 (2006).

27. Gou, X. \& Li, J. Paired receptor and coreceptor kinases perceive extracellular signals to control plant development. Plant Physiol. 182, 1667-1681 (2020).

28. Hosseini, S., Schmidt, E. D. L. \& Bakker, F. T. Leucine-rich repeat receptorlike kinase II phylogenetics reveals five main clades throughout the plant kingdom. Plant J. 103, 547-560 (2020)

29. Santiago, J. et al. Mechanistic insight into a peptide hormone signaling complex mediating floral organ abscission. elife. 5, e15075 (2016).

30. Franck, C. M., Westermann, J. \& Boisson-Dernier, A. Plant malectin-like receptor kinases: from cell wall integrity to immunity and beyond. Annu. Rev. Plant Biol. 69, 301-328 (2018).

31. Fletcher, J. C. Recent advances in Arabidopsis CLE peptide signaling. Trends Plant Sci. 25, 1005-1016 (2020).

32. Okuda, S. et al. Molecular mechanism for the recognition of sequencedivergent CIF peptides by the plant receptor kinases GSO1/SGN3 and GSO2. Proc. Natl Acad. Sci. USA 117, 2693-2703 (2020).

33. Bartels, S. \& Boller, T. Quo vadis, Pep? Plant elicitor peptides at the crossroads of immunity, stress, and development. J. Exp. Bot. 66, 5183-5193 (2015).

34. Whitewoods, C. D. Evolution of CLE peptide signalling. In Seminars in Cell and Developmental Biology https://doi.org/10.1016/j.semcdb.2020.04.022 (Academic press, 2020).

35. Pokhare, S. S. et al. Signatures of adaptation to a monocot host in the plant-parasitic cyst nematode Heterodera sacchari. Plant J. 103, 1263-1274 (2020).

36. Le Marquer, M., Ecard, G. B. \& Frei Dit Frey, N. Arbuscular mycorrhizal fungi possess a CLAVATA3/embryo surrounding region-related gene that positively regulates symbiosis. N. Phytol. 222, 1030-1042 (2019).

37. Bobay, B. G. et al. Solution NMR studies of the plant peptide hormone CEP inform function. FEBS Lett. 587, 3979-3985 (2013).

38. Zhang, X. et al. Nematode-encoded RALF peptide mimics facilitate parasitism of plants through the FERONIA receptor kinase. Mol. Plant 13, 1434-1454 (2020).

39. Wood, A. K. M., Walker, C., Lee, W. S., Urban, M. \& Hammond-Kosack, K. E. Functional evaluation of a homologue of plant rapid alkalinisation factor (RALF) peptides in Fusarium graminearum. Fungal Biol. 124, 753-765 (2020).

40. Masachis, S. et al. A fungal pathogen secretes plant alkalinizing peptides to increase infection. Nat. Microbiol. 1, 16043 (2016).
41. Fischer, I., Diévart, A., Droc, G., Dufayard, J. F. \& Chantret, N. Evolutionary dynamics of the leucine-rich repeat receptor-like kinase (LRR-RLK) subfamily in angiosperms. Plant Physiol. 170, 1595-1610 (2016).

42. Wang, Y. et al. Leucine-rich repeat receptor-like gene screen reveals that nicotiana RXEG1 regulates glycoside hydrolase 12 MAMP detection. Nat. Commun. 9, 594 (2018).

43. Zhang, J. et al. Receptor-like cytoplasmic kinases integrate signaling from multiple plant immune receptors and are targeted by a Pseudomonas syringae effector. Cell Host Microbe 7, 290-301 (2010).

44. Cutler, S. R., Ehrhardt, D. W., Griffitts, J. S. \& Somerville, C. R. Random GFP:: cDNA fusions enable visualization of subcellular structures in cells of Arabidopsis at a high frequency. Proc. Natl. Acad. Sci. USA 97, 3718-3723 (2000).

45. Nekrasov, V. et al. Control of the pattern-recognition receptor EFR by an ER protein complex in plant immunity. EMBO J. 28, 3428-3438 (2009).

46. Ranf, S. et al. Microbe-associated molecular pattern-induced calcium signaling requires the receptor-like cytoplasmic kinases, PBL1 and BIK1. BMC Plant Biol. 14, 374 (2014).

47. Clough, S. J. \& Bent, A. F. Floral dip: a simplified method for Agrobacteriummediated transformation of Arabidopsis thaliana. Plant J. 16, 735-743 (1998)

48. Earley, K. W. et al. Gateway-compatible vectors for plant functional genomics and proteomics. Plant J. 45, 616-629 (2006)

49. Hashimoto, Y., Zhang, S., Zhang, S., Chen, Y. R. \& Blissard, G. W. Correction: BTI-Tnao38, a new cell line derived from Trichoplusia ni, is permissive for AcMNPV infection and produces high levels of recombinant proteins. BMC Biotechnol. 12, 12 (2012).

50. Kumar, S., Stecher, G., Suleski, M. \& Hedges, S. B. Timetree: a resource for timelines, timetrees, and divergence times. Mol. Biol. Evol. 34, 1812-1819 (2017).

51. Felix, G., Duran, J. D., Volko, S. \& Boller, T. Plants have a sensitive perception system for the most conserved domain of bacterial flagellin. Plant J. 18, 265-276 (1999).

52. Huffaker, A., Pearce, G. \& Ryan, C. A. An endogenous peptide signal in Arabidopsis activates components of the innate immune response. Proc. Natl. Acad. Sci. USA 103, 10098-10103 (2006).

53. Kunze, $\mathrm{G}$. et al. The $\mathrm{N}$ terminus of bacterial elongation factor $\mathrm{Tu}$ elicits innate immunity in Arabidopsis plants. Plant Cell 16, 3496-3507 (2004)

\section{Acknowledgements}

We thank Justin Lee, Jean-Pierre Renou, Jen Sheen, Wei-Cai Yang, and Yuelin Zhang for providing published materials, the John Innes Centre Horticultural Services for plant care, and all past and current members of the Zipfel and Santiago groups for technical help and fruitful discussions. Nicholas Talbot is acknowledged for hosting J.R. for part of this study. This work was supported by the European Research Council under the Grant Agreements no. 773153 and no. 716358 (grant "IMMUNO-PEPTALK" to C.Z. and gran "WallWatchers" to J.S., respectively), The Gatsby Charitable Foundation (to C.Z.), the University of Zürich (to C.Z.), the Swiss National Science Foundation grants no. 31003A_182625 (to C.Z.) and no. 31003A_173101 (to J.S.), and the Fondation Philanthropique Famille Sandoz (to J.S.).

\section{Author contributions}

Conceptualization, C.Z. and J.S.; methodology, C.Z. and J.S.; investigation, J.R., H.J., S.M. and F.B.; writing-original draft, J.R. and C.Z.; writing-review \& editing, all authors; funding acquisition, C.Z. and J.S.; resources, F.B.; supervision, C.Z. and J.S.

\section{Competing interests}

A provisional patent application on the use of SCOOPs and MIK2 in crop protection has been filed. Patent applicant: authors; the name of inventors: C.Z., J.R. and H.Y.; application number: 070294.0168; Status of application: pending. The remaining authors declare no competing interests.

\section{Additional information}

Supplementary information The online version contains supplementary material available at https://doi.org/10.1038/s41467-021-20932-y.

Correspondence and requests for materials should be addressed to C.Z.

Peer review information Nature Communications thanks the anonymous reviewers for their contribution to the peer review of this work. Peer reviewer reports are available.

Reprints and permission information is available at http://www.nature.com/reprints

Publisher's note Springer Nature remains neutral with regard to jurisdictional claims in published maps and institutional affiliations. 
(c) (i) Open Access This article is licensed under a Creative Commons Attribution 4.0 International License, which permits use, sharing, adaptation, distribution and reproduction in any medium or format, as long as you give appropriate credit to the original author(s) and the source, provide a link to the Creative Commons license, and indicate if changes were made. The images or other third party material in this article are included in the article's Creative Commons license, unless indicated otherwise in a credit line to the material. If material is not included in the article's Creative Commons license and your intended use is not permitted by statutory regulation or exceeds the permitted use, you will need to obtain permission directly from the copyright holder. To view a copy of this license, visit http://creativecommons.org/ licenses/by/4.0/.

(C) The Author(s) 2021 